\section{Structure and Diamagnetic Susceptibility of Cyclo-octatetraene}

According to Pascal's empirical formulæ ${ }^{1}$, if cyclo-octatetraene has a structure corresponding with a cyclic system of conjugated double and single bonds, which may be puckered, the molecular susceptibility to be expected is $-50.2 \times 10^{-6}$ c.G.S. On the other hand, if it contains eight 'aromatic' carbon atoms with eight pooled electrons, the molecular susceptibility to be expected would be $-73.4 \times 10^{-6}$ c.G.S. This characteristic increase in diamagnetic susceptibility when a pool of 'aromatic' electrons is present ${ }^{2}$ makes a useful index of aromatic character. In the case of cyclo-octatetraene, recently published data for the heat of formation ${ }^{3}$ lead to a calculated resonance energy of $\mathbf{2 5 . 4} \mathrm{kcal} / \mathrm{mole}$. Although this resonance energy is smaller than in the case of benzene (approximately $39 \mathrm{kcal} . / \mathrm{mol} \theta)^{5}$, it is sufficiently high to leave some doubt as to whether cyclooctatetraene may have its structure stabilized by the formation of an aromatic pool of electrons. Optical evidence ${ }^{4}$ is not quite decisive on this point. Measurements have now been made on the magnetic susceptibility of cyclo-octatetraene, to test for an aromatic pool of electrons by the enhanced diamagnetism.

Details of these measurements are being published elsewhere; but the main result is that the molecular susceptibility of liquid cyclo-octatetraene at $20^{\circ} \mathrm{C}$. is $-51.9 \times 10^{-6}$. This appears to indicate that the structure corresponds primarily with a cyclic system of conjugated double and single bonds, and not with an aromatic pool.

At the same time, the colour of solid cyclo-octatetraene appears to change from yellow to white at low temperatures. This may be associated with some change in bond structure, such as the disappearance of small amounts of a di-radical form of cyclo-octatetraene on cooling, and further experiments are in hand on solid cyclo-octatetraene at low temperatures.

Thanks are due to Messrs I.C.I. for gifts of cyclooctatetraene and related compounds.

\section{R. C. PINK \\ A. R. UBBELOHDE}

Chemistry Department,

Queen's University, Belfast.

${ }^{1}$ Cf. Selwood, "Magneto-chemistry" (Interscience, 1943).

\& Ubbelohde, Nature, 132, 1002 (1933). Krishnan and Banerjee, Phil. Trans. Roy. Soc., A, 234, 278 (1935). London, J. de Phys., 8, 397 (1937).

'B.I.O.S. Report, N.137, Item 22 (1945),

- Flett, Cave, Vago and Thompson, Nature, 159, 739 (1947). Lippin-

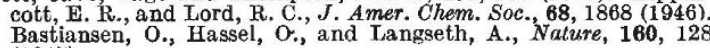
(1947).

s Pauling, "Nature of the Chemical Bond" (1940), p. 134.

\section{The Isomeric Trithioaldehydes}

Schönberg and Barakat contend ${ }^{x}$ that the pairs of isomeric trithioaldehydes ( $\mathrm{I}$ ) hitherto regarded as having their substituents in cis and trans positions with respect to the ring are, in fact, relatively stable 'boat' and 'chair' ring isomerides. These authors suggest that the trithioaldehydes are resonance hybrids of forms such as I and II
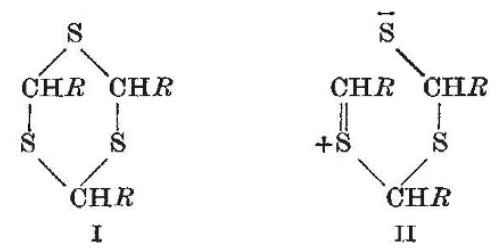

implying that this resonance would account for some special stability of the two puckered forms.

This view could not be accepted unless it were shown that the postulated resonance would, in fact, stabilize each of the puckered ring forms, as com. pared with any possible intermediate configuration, so as to introduce an energy barrier of sufficient magnitude to prevent a rapid interconversion such as occurs with the corresponding forms of cyclohexarie. But the opposite conclusion appears more probable: for it is clear that the resonance would affect equally all the bonds in the ring, imparting to each some degree of double-bond character. This should stabilize a planar or nearly planar form rather than the 'boat' and 'chair' configurations.

Apart from this, the authors appear to have overlooked several significant points. (1) Why, if their suggestion is correct, do not two forms of the parent trimethylene trisulphide exist? (2) The authors' hypothesis requires more than two isomerides of the trithioaldehydes and they refer to various reports of third isomerides. The case of the trithioacetaldehydes is one of the most carefully investigated, and the evidence that the supposed third 'isomeride' is a mixture of the other two is conclusive ${ }^{2}$. (3) Chattaway and Kellett ${ }^{3}$ succeeded in oxidizing the two trithioacetaldehydes to three isomeric monosulphones. This result is exactly that required by the classical view of the isomerism and is strong evidence of its correctness. (4) The catalytic effect of iodine in causing interconversion of the isomeric trithioaldehydes is not surprising. The ring carbon atoms are each attached to two sulphur atoms, and a reversible rupture of a carbon-sulphur bond in these circumstances is reasonable. Any such reaction would, however, be retarded by the presence of a sulphone group, and, in fact, Chattaway's isomeric sulphones were found not to be interconvertible by catalysis.

There is thus no reason to doubt that the isomerism of the trithioaldehydes is of the cis - trans type.

Government Laboratory,

G. M. BennetT

London, W.C.2.

Aug. 7.

${ }^{1} \mathrm{~J}$. Chem. Soc., 693 (1947).

${ }^{2}$ Bell, Bennett, and Hock, J. Chem. Soc., 1462 (1929).

${ }^{3}$ J. Chem. Soc., 1352 (1930).

\section{Isolation of a Quadrivalent Rhodium Complex}

THE only compound of quadrivalent rhodium described in the literature is the green, hydrated oxide obtained in the impure form by passing chlorine into a solution of sodium hexachlor-rhodate(III), $\left(\mathrm{Na}_{3} \mathrm{RhCl}_{6}\right)$, containing excess sodium hydroxide ${ }^{1}$. Grube and $\mathrm{Gu}^{2}$ obtained greenish-violet coloured solutions by oxidizing anodically rhodium salts in sulphuric, nitric and perchloric acids. Although no solid products were isolated, the authors deduced from potentiometric titrations that these solutions contained some quadrivalent and hexavalent rhodium. The redox potential of the trivalent-quadrivalent couple was estimated at +1.40 volts.

Although the most characteristic compounds of the quadrivalent state among the platinum metals are the hexahalogen complexes, the above potential leads to the conclusion that any complexes of quadrivalent rhodium containing halogens, except fluorine, would be highly unstable in aqueous solution. In conformity with the large size of the cation, the 